\title{
New Estimator for AR (1) Model with Missing Observations
}

\author{
Mohamed Khalifa Ahmed Issa \\ Assistant Professor of Statistics, Department of Statistics \\ The Higher Institute of Cooperative and Managerial Studies, Egypt \\ mk_mk2229@yahoo.com
}

\begin{abstract}
In this paper, new form of the parameters of $A R(1)$ with constant term with missing observations has been derived by using Ordinary Least Squares (OLS) method, Also, the properties of OLS estimator are discussed, moreover, an extension of Youssef [18] has been suggested for AR(1) with constant with missing observations. A comparative study between $(O L S)$, Yule-Walker $(Y W)$ and modification of the ordinary least squares (MOLS) is considered in the case of stationary and near unit root time series, using Monte Carlo simulation.
\end{abstract}

Keywords and phrases: near unit root, modification ordinary least square estimator, AR (1) Model with constant with missing observations, Yule-Walker estimator

\section{Introduction}

A survey of methodologies for handling missing-data problems with emphasis on likelihood methods and the expectation-maximization (EM) algorithm is presented by Little and Rubin [11]. Parzen [12], a time series with missing observations can be regarded as an amplitude modulated version of the original time series, i.e

$$
{ }_{k}=a_{k} x_{k} \quad k=1,2, \ldots, n
$$

Where $x_{k}$ is assumed to be defined for all time and $a_{k}$ represented the state of observation.

$$
a_{k}= \begin{cases}1 & \text { if } x_{k} \text { is observed } \\ 0 & \text { if } x_{k} \text { is missing }\end{cases}
$$

and $y_{k}$ represents the observed value of $x_{k}$, if $a_{k}=1$ otherwise $y_{k}$ will be equal to zero if $x_{k}$ is missing. In practice, missing values may occur regularly or randomly. Jones [10] and Parzen [12] considered the case of periodic sampling where the observed data consist of repeated in two groups, the first group consecutive observations followed by second group missed observations. Following Parzen [11] concentrated on the analysis of irregularly observed time series and contains theoretical and practical contributions. In contrast, Scheinok [15] and Bloomfield [3] investigated the case where $x_{k}$ is observed 
when a "success" is achieved on a Bernoulli trial. These references concentrate on nonparametric spectral analysis of time series with missing values, while a parametric approach is considered by Dunsmuir and Robinson [5]. In the time domain, asymptotic properties of non-parametric estimators of autocovariances and autocorrelations of amplitude modulated time series are established by Dunsmuir and Robinson [6] and Yajima and Nishino [16]. These results can be used to build Yule-Walker type estimators for an AR process with missing observations.

The literatures have several approaches to estimate AR model with missing observations. Abdelwahab [1] extended the estimator of Dunsmuir and Robinson [4] of $\operatorname{AR}(1)$ with constant term with missing observations be using Yule-Walker method when $|\rho|<1$ is given by

$$
\hat{\rho}_{Y W}=\frac{\sum_{k=2}^{n} z_{k} z_{k-1} / \sum_{k=2}^{n} a_{k} a_{k-1}}{\sum_{k=1}^{n-1} z_{k-1}^{2} / \sum_{k=1}^{n-1} a_{k}}
$$

Where $Z_{k}={ }_{k}-\mu$ and $Z_{k-1}={ }_{k-1}-\mu$

El-sayed et al. [7] derived OLS estimator for AR (1) without constant term in case of missing values. In addition, the properties of the estimator's linearity and unbiased are discussed. Saadatmand, et al [14] considered estimation of a missing value for $A R$ (1) with exponential innovations and compared two methods of estimation of the missing value, with respect to Pitman's measure of closeness. AbdelWahab and Issa [2] derived a general form of the mean and variance of AR (P) model with missing observations, and a special cases also introduced. In [9] they derived OLS estimator for AR (1) panel data model with missing data, which can be considered as an extension of El-Sayed, et al [7]. In addition Enany, et al. [8] introduced a closed form estimator for $\rho$ in case of missing observations using maximum likelihood (ML) of $\mathrm{AR}(1)$ model without constant term with missing observations when $y_{0}$ is random. The rest of this paper is organized as follows: section (2) the model and its assumptions has been introduced and alternative estimators for AR(1) model with constant term and missing observations has been obtained. Moreover, the properties of the estimator are investigated. In section (3), simulation studies are carried out to compare between different estimators YW estimator, and the proposed estimators (OLS and MOLS). Finally, in section (4), conclusion of the theory and simulation study has been presented.

\section{Model and Alternative Estimators}

Parzen [12] introduced the time series model with missing observations as a specific case of an amplitude modulated stationary process. He observed that the data $\left(x_{1}, x_{2}, x_{3}, \ldots, x_{p}\right)$ can be represented as,

$$
x_{k}=\eta+\rho x_{k-1}+\varepsilon_{k}, \quad k=1,2, \ldots, n
$$


To specify the model in (3), the following assumptions are imposed.

a) The unknown parameters $\rho$ and $\eta$ are constrained to $|\rho|<1$ for stationarity.

b) ${ }_{0}$ is fixed and when $n \rightarrow \infty$ the effect of $\varepsilon_{1}$ will be negligible and so it will tends to zero.

c) $\varepsilon_{k}$ is (i.i.d) with a Gaussian distribution with mean 0 and variances $\sigma_{\varepsilon}^{2}$ and the fourth moment of $\varepsilon_{\mathrm{k}}$ exists.

d) $E\left({ }_{k-1}, \varepsilon_{k}\right)=0$, the independent variable are predetermined in the sense that they are orthogonal to the contemporaneous error term for every $\mathrm{k}=2,3, \ldots, \mathrm{n}$.

e) $E\left(\varepsilon_{i}, \varepsilon_{k}\right)=0$

f) $a_{k-1} a_{k}=1$ (this assumption stated by Takeuchi [16])

\subsection{Least Square Estimator of AR (1) Model}

Pre multiply $\mathrm{a}_{\mathrm{k}}$ of both sides of equation (3), to get,

$$
{ }_{k}=\eta a_{k}+\rho a_{k-1}+a_{k} \varepsilon_{k}
$$

The least square estimator for $\eta$ and $\rho$, can be obtained by regressing, including an intercept, $\mathrm{y}_{\mathrm{k}}$ on $\mathrm{y}_{\mathrm{k}-1}$ as in model (1). Let, $\mathrm{Q}$ be the sum of squares of the random variables of model (4) of the estimated residuals:

$$
Q_{o l s}=\sum_{k=2}^{n} a_{k} \varepsilon_{k}^{2}=\sum_{k=2}^{n}\left[y_{k}-\eta a_{k}-\rho a_{k} k-1\right]^{2}
$$

By differentiating equation (5) with respect to $\rho, \eta$ and set to zero then,

$$
\begin{aligned}
& \hat{\rho}_{\text {ols }}=\frac{\sum_{k=2}^{n} a_{k} \quad k \quad k-1}{-\hat{\eta} \sum_{k=2}^{n} a_{k} \quad k-1} \\
& \hat{\eta}_{o l s}=\frac{\sum_{k=2}^{n} a_{k} k}{n-c-1}-\frac{\hat{\rho}_{o l s} \sum_{k=2}^{n} a_{k k-1}}{n-c-1}
\end{aligned}
$$

Where $\mathrm{n}$ is sample size and $\mathrm{c}$ is number of missing values. By substituting the value $\hat{\eta}_{\text {ols }}$ of equation (7), to get

$$
\hat{\rho}_{\text {ols }}=\frac{\sum_{k=2}^{n} a_{k} \quad k \quad k-1}{-\frac{\sum_{k=2}^{n} a_{k \quad k} \sum_{k=2}^{n} a_{k} \quad k-1}{n-c-1}}
$$

\section{- Properties of the Least Square Estimator}

Some properties of the OLS method for the estimator of AR(1) with missing observations model with constant will be discussed. 


\section{- The linearity of $\rho$}

By taking $\sum_{\mathrm{k}=2}^{\mathrm{n}} \mathrm{a}_{\mathrm{k}} \mathrm{y}_{\mathrm{k}}$ as a common factor of the numerator

$$
\hat{\rho}_{\text {ols }}=\frac{\sum_{k=2}^{n} a_{k \quad k}\left(y_{k-1}-\frac{\sum_{k=2}^{n} a_{k k-1}}{n-c-1}\right)}{\sum_{k=2}^{n} a_{k}{ }_{k-1}^{2}-\frac{\left(\sum_{k=2}^{n} a_{k-1}\right)^{2}}{n-c-1}}
$$

From equation (9), it is easy to verify that $\hat{\rho}_{\text {ols }}$ can be rewritten as liner form

$$
\hat{\rho}_{\text {ols }}=\sum_{k=2}^{n} M_{k}
$$

Where

$$
M_{k}=a_{k}\left(y_{k-1}-\frac{\sum_{k=2}^{n} a_{k} k-1}{n-c-1}\right)\left(\sum_{k=2}^{n} a_{k}{ }_{k-1}^{2}-\frac{\left(\sum_{k=2}^{n} a_{k} \quad k-1\right)^{2}}{n-c-1}\right)^{-1}
$$

To prove the unbiasedness and variance property of $\hat{\rho}_{\text {ols }}$ the following lime need to establish.

\section{Lemma (1):}

(i) $\sum_{k=2}^{n} a_{k} M_{k}=0$

(ii) $\sum_{k=2}^{n} a_{k} M_{k} \quad k-1=1$

(iii) $\sum_{k=2}^{n} a_{k} M_{k}^{2}=1 /\left[\sum_{k=2}^{n} a_{k}{ }_{k-1}^{2}-\frac{\left(\sum_{k=2}^{n} a_{k} y_{k-1}\right)^{2}}{n-c-1}\right]$

\section{Proof}

(i) Since,

$$
\begin{aligned}
& \sum_{k=2}^{n} a_{k} M_{k}=\sum_{k=2}^{n} a_{k}\left(y_{k-1}-\frac{\sum_{k=2}^{n} a_{k} k-1}{n-c-1}\right)\left(\sum_{k=2}^{n} a_{k}{ }_{k-1}^{2}-\frac{\left(\sum_{k=2}^{n} a_{k} k-1\right)^{2}}{n-c-1}\right)^{-1} \\
& =\left(\sum_{k=2}^{n} \boldsymbol{a}_{k} \quad k-1-\frac{\sum_{k=2}^{n} \boldsymbol{a}_{k} \sum_{k=2}^{n} a_{k} k-1}{n-c-1}\right)\left(\sum_{k=2}^{n} a_{k}{ }_{k-1}^{2}-\frac{\left(\sum_{k=2}^{n} a_{k} k-1\right)^{2}}{n-c-1}\right)^{-1}
\end{aligned}
$$

Since, $\sum_{k=2}^{n} a_{k}=n-c-1$, then

$$
\sum_{k=2}^{n} a_{k} M_{k}=0
$$

(ii) Since,

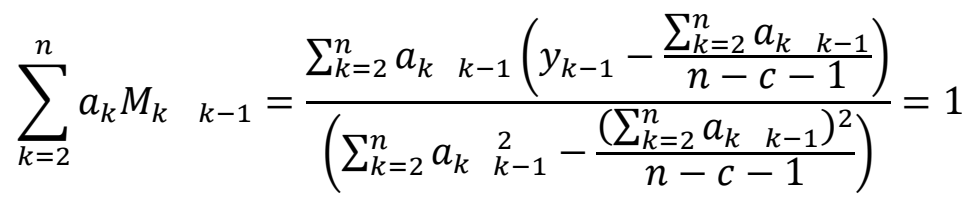


(iii) Since,

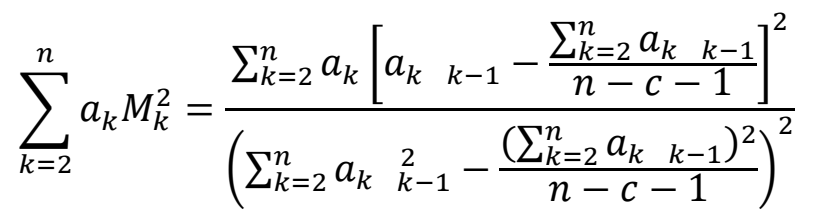

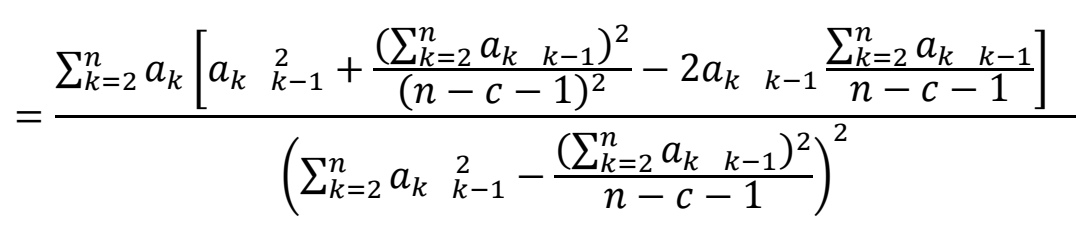

$$
\begin{aligned}
& =\frac{\left[\sum_{k=2}^{n} a_{k}{ }_{k-1}^{2}+\frac{(n-c-1)\left(\sum_{k=2}^{n} a_{k} k-1\right)^{2}}{(n-c-1)^{2}}-2 \sum_{k=2}^{n} a_{k} \quad k-1 \frac{\left(\sum_{k=2}^{n} a_{k k-1}\right)}{n-c-1}\right]}{\left(\sum_{k=2}^{n} a_{k}{ }_{k-1}^{2}-\frac{\left(\sum_{k=2}^{n} a_{k} k-1\right)^{2}}{n-c-1}\right)^{2}} \\
& =\frac{\left[\sum_{k=2}^{n} a_{k}{ }_{k-1}^{2}+\frac{\left(\sum_{k=2}^{n} a_{k} k-1\right)^{2}}{(n-c-1)}-2 \frac{\left(\sum_{k=2}^{n} a_{k} k-1\right)^{2}}{n-c-1}\right]}{\left(\sum_{k=2}^{n} a_{k}{ }_{k-1}^{2}-\frac{\left(\sum_{k=2}^{n} a_{k} k-1\right)^{2}}{n-c-1}\right)^{2}}
\end{aligned}
$$

By simplifying equation (11) $\sum_{k=2}^{n} a_{k} M_{k}^{2}$ has its form

\section{- The Unbiasedness of $\widehat{\boldsymbol{\rho}}_{\text {ols }}$}

By substituting ${ }_{k}$ of equation (4) in equation (10), to get

$$
\hat{\rho}_{\text {ols }}=\eta \sum_{k=2}^{n} a_{k} M_{k}+\rho \sum_{k=2}^{n} a_{k} M_{k} \quad k-1+\sum_{k=2}^{n} M_{k} a_{k} \varepsilon_{k}
$$

From result of lemma (1), then

$$
\hat{\rho}_{\text {ols }}=\rho+\sum_{k=2}^{n} M_{k} a_{k} \varepsilon_{k}
$$

By taking the expectation of equation (13), so, $\hat{\rho}_{\text {ols }}$ is unbiased estimator of $\rho$

Not: $\hat{\eta}_{\text {ols }}$ is unbiased estimator of $\eta$ (The proof is omitted).

\section{- The Variance of the Estimators $\widehat{\boldsymbol{\rho}}_{\text {ols }}$}

The variance of the (OLS) estimator can be obtained as follows:

$$
\operatorname{var}\left(\hat{\rho}_{o L S}\right)=E\left[\hat{\rho}_{o L S}-\rho\right]^{2}
$$

By substituting from (13) in equation (14), to get;

$$
\operatorname{var}\left(\hat{\rho}_{o L S}\right)=E\left[\sum_{k=2}^{n} a_{k} M_{k} \varepsilon_{k}\right]^{2}
$$




$$
\operatorname{var}\left(\hat{\rho}_{o L S}\right)=E\left[\sum_{k=2}^{n} a_{k} M_{k}^{2} \varepsilon_{k}^{2}+\sum_{k=2}^{n} \sum_{\substack{i=2 \\ i \neq k}} \alpha_{k} \alpha_{i} M_{k} M_{i} \varepsilon_{k} \varepsilon_{i}\right]
$$

By taking the expectation in (15), to get;

$$
\operatorname{var}\left(\hat{\rho}_{o l s}\right)=\frac{\hat{\sigma}_{o l s}^{2}}{\sum_{k=2}^{n} a_{k}{ }_{k-1}^{2}-\frac{\left(\sum_{k=2}^{n} a_{k} k-1\right)^{2}}{n-c-1}}
$$

\subsection{Modification Least Square Estimator of AR (1)}

In this section, we suggested a modification of Youssef (2006) in case of missing observations, is given by:

$$
\hat{\rho}_{M O L S}=\hat{\rho}_{o l s}+c\left(\hat{\varphi}_{o l s}\right)\left[\widehat{v a r}\left(\hat{\rho}_{o l s}\right)\right]^{\frac{1}{2}}
$$

and an estimator of

Where

$$
\hat{\eta}_{\text {mols }}=\bar{y}_{(0)}-\hat{\rho}_{\text {ols }} \bar{y}_{(-1)}
$$

$$
c\left(\hat{\varphi}_{\text {ols }}\right)= \begin{cases}0 & \text { if } \hat{\varphi}_{\text {ols }} \geq-7.1, \\ 0.062222\left(\hat{\varphi}_{\text {ols }}+7.1\right)^{2} & \text { if }-7.1 \leq \hat{\varphi}_{\text {ols }}<-3.6, \\ 1.71-0.062222\left(\hat{\varphi}_{\text {ols }}+0.10\right)^{2} & \text { if }-3.6 \leq \hat{\varphi}_{\text {ols }} \leq 3.4 \\ 0.062222\left(\hat{\varphi}_{\text {ols }}-6.90\right)^{2} & \text { if } 3.4<\hat{\varphi}_{\text {ols }} \leq 6.9 \\ 0 & \text { if } \hat{\varphi}_{\text {ols }}>6.9\end{cases}
$$

and

$$
\hat{\varphi}_{o l s}=\left(\operatorname{var}\left(\hat{\rho}_{o l s}\right)\right)^{\frac{-1}{2}}\left(\hat{\rho}_{o l s}-1\right)
$$

We choose the function $c\left(\hat{\varphi}_{\text {ols }}\right)$ to be a smooth function of the statistic $\hat{\varphi}_{\text {ols }}$ to remove fluctuations in an ordered series, so that the result shall be smooth in the sense that the first-order differences errors must be regular and small.

\section{Simulations Study}

Here we analyse the finite sample behavior of estimators for OLS, YW and MOLS are defined by Monte Carlo simulations. The random errors is assumed to be standard normal distribution. A Comparison between (OLS), (YW) and (MOLS) estimators for AR (1) is presented using MSE as criteria. The setting of model and the result of the simulation study are discussed. To perform the simulation, the model is needed to construct as follows:

1- AR (1) model with constant is generated. The errors are generated $\sim \operatorname{IIDN}(0,1)$, and the autoregressive parameter $\rho$ is chosen to be $(0.4,0.7,95$ and 0.99$)$ and $\eta$ is chosen to be (0,2 and 5).

2- The values of sample size $\mathrm{n}$ are equal to $20,50,100$ and 250 to represent small, medium and large samples of time series. 
3- To investigate the resistance of the proposed estimators, we randomly generate different percentages of missing values equals to [(5 to 10), (15 to 20) and (23 to 30)] based on MSE as a criteria of comparison.

From tables (1 to 3) MOLS has better performance for small, medium and large sample size whatever the percentages of missing values and the values of $\rho$ (Specially for the case of nearing unit root) compering with the other methods. While for small simple size ( $n=20$ and $\rho=0.99)$ the MSE of (YW) method is inflated otherwise YW and OLS are almost for large sample size excepted for case of nearing unit root when $(\rho=0.99)$ the MSE of (YW) methods is decrease as the sample size increase. Also, there is a positive correlation between the percentage of missing values and the value of the MSE whatever methods of estimation.

\section{Conclusion}

In this article, a new form of the estimator of the parameters of $\operatorname{AR}(1)$ with constant term with missing observations has been derived by using OLS and MOLS. Also, the linearity, the unbiasedness and the variance of the estimated parameters has been proved for OLS method. Also, a modification of the ordinary least square estimator for AR (1) with constant term with missing observations has been suggested. Moreover, the results of Monte Carlo simulation have been approved good results for MOLS compering with the other methods.

\section{Appendix}

Table 1: MSE of AR (1) with constant when presenting missing from (5 to 10) \%

\begin{tabular}{|c|c|c|c|c|c|}
\hline \multirow{2}{*}{$\mathbf{n}$} & \multirow[b]{2}{*}{$\eta$} & \multirow[b]{2}{*}{$\rho$} & \multicolumn{3}{|c|}{ Methods } \\
\hline & & & OLS & MOLS & YW \\
\hline \multirow{12}{*}{20} & \multirow{4}{*}{$\mathbf{0}$} & 0.4 & 0.968393 & 0.1805806 & 0.9716754 \\
\hline & & 0.7 & 1.032027 & 0.1298812 & 1.044211 \\
\hline & & 0.95 & 1.604699 & 0.336904 & 2.477449 \\
\hline & & 0.99 & 10.76233 & 1.278834 & 101.0191 \\
\hline & \multirow{4}{*}{2} & 0.4 & 0.963473 & 0.1809134 & 0.966687 \\
\hline & & 0.7 & 1.035475 & 0.1323727 & 1.047395 \\
\hline & & 0.95 & 1.580166 & 0.3402403 & 2.29778 \\
\hline & & 0.99 & 6.792754 & 1.096909 & 101.0087 \\
\hline & \multirow{4}{*}{5} & 0.4 & 0.982783 & 0.5999709 & 1.006373 \\
\hline & & 0.7 & 1.17732 & 0.4441973 & 1.254056 \\
\hline & & 0.95 & 2.096177 & 0.658379 & 10.00071 \\
\hline & & 0.99 & 3.809973 & 1.066639 & 380.3538 \\
\hline \multirow{5}{*}{50} & \multirow{4}{*}{$\mathbf{0}$} & 0.4 & 0.9946356 & 0.05153484 & 0.9955089 \\
\hline & & 0.7 & 1.047994 & 0.04088866 & 1.052115 \\
\hline & & 0.95 & 1.54701 & 0.04825183 & 1.597877 \\
\hline & & 0.99 & 2.867574 & 0.2552623 & 4.526094 \\
\hline & 2 & 0.4 & 0.9939973 & 0.05131506 & 0.9948659 \\
\hline
\end{tabular}




\begin{tabular}{|c|c|c|c|c|c|}
\hline \multirow{9}{*}{$\mathbf{n}$} & \multirow{5}{*}{$\eta$} & \multirow[b]{2}{*}{$\rho$} & \multicolumn{3}{|c|}{ Methods } \\
\hline & & & OLS & MOLS & YW \\
\hline & & 0.7 & 1.046383 & 0.04036824 & 1.050568 \\
\hline & & 0.95 & 1.555653 & 0.04707399 & 1.604129 \\
\hline & & 0.99 & 2.824367 & 0.2520745 & 4.706201 \\
\hline & \multirow{4}{*}{5} & 0.4 & 0.9906181 & 0.05159136 & 0.9915005 \\
\hline & & 0.7 & 1.051061 & 0.04020128 & 1.055237 \\
\hline & & 0.95 & 1.55386 & 0.04803618 & 1.602607 \\
\hline & & 0.99 & 2.853917 & 0.2503087 & 4.203451 \\
\hline \multirow{12}{*}{100} & \multirow{4}{*}{$\mathbf{0}$} & 0.4 & 1.003708 & 0.01998433 & 1.004219 \\
\hline & & 0.7 & 1.067184 & 0.01854758 & 1.069772 \\
\hline & & 0.95 & 1.697259 & 0.01453212 & 1.744177 \\
\hline & & 0.99 & 3.579322 & 0.1028003 & 4.276545 \\
\hline & & 0.4 & 1.005733 & 0.01972462 & 1.006243 \\
\hline & 2 & 0.7 & 1.067159 & 0.01835664 & 1.069733 \\
\hline & 2 & 0.95 & 1.704838 & 0.01457261 & 1.750174 \\
\hline & & 0.99 & 3.615873 & 0.1038923 & 4.320035 \\
\hline & & 0.4 & 1.005458 & 0.01989451 & 1.005963 \\
\hline & & 0.7 & 1.068698 & 0.01858591 & 1.071233 \\
\hline & 5 & 0.95 & 1.691083 & 0.01373967 & 1.736293 \\
\hline & & 0.99 & 3.649369 & 0.1081818 & 4.3734 \\
\hline & & 0.4 & 1.01063 & 0.006324722 & 1.010818 \\
\hline & 0 & 0.7 & 1.066909 & 0.004926175 & 1.067891 \\
\hline & $\mathbf{0}$ & 0.95 & 1.68105 & 0.002955617 & 1.697834 \\
\hline & & 0.99 & 4.162772 & 0.0239558 & 34.61238 \\
\hline & & 0.4 & 1.052456 & 0.01487625 & 1.053558 \\
\hline & & 0.7 & 1.282189 & 0.01576271 & 1.2886 \\
\hline 250 & 2 & 0.95 & 3.619094 & 0.01543403 & 3.81975 \\
\hline & & 0.99 & 10.9264 & 0.1054635 & 15.41834 \\
\hline & & 0.4 & 1.007877 & 0.00658123 & 1.008063 \\
\hline & 5 & 0.7 & 1.068466 & 0.00475400 & 1.069423 \\
\hline & 5 & 0.95 & 1.671939 & 0.00288914 & 1.688428 \\
\hline & & 0.99 & 4.134925 & 0.0234227 & 4.567435 \\
\hline
\end{tabular}


Table 2: MSE of AR (1) with constant when presenting missing from (15 to 20) \%

\begin{tabular}{|c|c|c|c|c|c|}
\hline \multirow[b]{2}{*}{$\mathbf{n}$} & \multirow[b]{2}{*}{$\eta$} & \multirow[b]{2}{*}{$\rho$} & \multicolumn{3}{|c|}{ Methods } \\
\hline & & & OLS & MOLS & YW \\
\hline \multirow{12}{*}{20} & \multirow{4}{*}{$\mathbf{0}$} & 0.4 & 0.9749133 & 0.2451357 & 0.981976 \\
\hline & & 0.7 & 1.102778 & 0.1841462 & 1.128937 \\
\hline & & 0.95 & 1.81887 & 0.413793 & 74.11254 \\
\hline & & 0.99 & 3.448981 & 0.886506 & 177.314 \\
\hline & \multirow{4}{*}{2} & 0.4 & 0.973123 & 0.2524433 & 0.9802957 \\
\hline & & 0.7 & 1.096779 & 0.1867491 & 1.12212 \\
\hline & & 0.95 & 1.805127 & 0.4196555 & 3.93168 \\
\hline & & 0.99 & 3.506999 & 0.8957062 & 187.4455 \\
\hline & \multirow{4}{*}{5} & 0.4 & 0.9795613 & 0.2480053 & 0.9866971 \\
\hline & & 0.7 & 1.100043 & 0.1889251 & 1.126146 \\
\hline & & 0.95 & 1.812485 & 0.417819 & 4.289986 \\
\hline & & 0.99 & 3.520894 & 0.9008005 & 202.2944 \\
\hline \multirow{12}{*}{50} & \multirow{4}{*}{$\mathbf{0}$} & 0.4 & 1.004058 & 0.07651163 & 1.00635 \\
\hline & & 0.7 & 1.135946 & 0.05426383 & 1.147093 \\
\hline & & 0.95 & 2.16274 & 0.09801211 & 2.396821 \\
\hline & & 0.99 & 3.950891 & 0.3643709 & 9.264502 \\
\hline & \multirow{4}{*}{2} & 0.4 & 1.007852 & 0.07405498 & 1.010127 \\
\hline & & 0.7 & 1.133281 & 0.05547938 & 1.144867 \\
\hline & & 0.95 & 2.154456 & 0.09723346 & 2.381065 \\
\hline & & 0.99 & 3.918684 & 0.357317 & 9.800973 \\
\hline & \multirow{4}{*}{5} & 0.4 & 1.007661 & 0.07536211 & 1.009891 \\
\hline & & 0.7 & 1.135624 & 0.05551519 & 1.146992 \\
\hline & & 0.95 & 2.159953 & 0.09605557 & 2.396225 \\
\hline & & 0.99 & 3.917036 & 0.3599319 & 10.57138 \\
\hline \multirow{12}{*}{100} & \multirow{4}{*}{$\mathbf{0}$} & 0.4 & 1.024281 & 0.02889199 & 1.025523 \\
\hline & & 0.7 & 1.155633 & 0.02658009 & 1.162268 \\
\hline & & 0.95 & 2.461991 & 0.03391199 & 2.638604 \\
\hline & & 0.99 & 5.540116 & 0.185878 & 8.134825 \\
\hline & \multirow{4}{*}{2} & 0.4 & 1.024384 & 0.02814007 & 1.025602 \\
\hline & & 0.7 & 1.158659 & 0.02678125 & 1.165434 \\
\hline & & 0.95 & 2.4726 & 0.03179676 & 2.64757 \\
\hline & & 0.99 & 5.545234 & 0.1917081 & 8.20409 \\
\hline & \multirow{4}{*}{5} & 0.4 & 1.023849 & 0.02768167 & 1.025095 \\
\hline & & 0.7 & 1.157484 & 0.02690667 & 1.164363 \\
\hline & & 0.95 & 2.458909 & 0.03305783 & 2.637638 \\
\hline & & 0.99 & 5.542934 & 0.1883303 & 8.190199 \\
\hline
\end{tabular}




\begin{tabular}{|c|c|c|c|c|c|}
\hline \multirow[b]{2}{*}{$\mathbf{n}$} & \multirow[b]{2}{*}{$\eta$} & \multirow[b]{2}{*}{$\boldsymbol{\rho}$} & \multicolumn{3}{|c|}{ Methods } \\
\hline & & & OLS & MOLS & YW \\
\hline \multirow{12}{*}{250} & \multirow{4}{*}{$\mathbf{0}$} & 0.4 & 1.027401 & 0.008150639 & 1.027898 \\
\hline & & 0.7 & 1.161601 & 0.006983699 & 1.164324 \\
\hline & & 0.95 & 2.544047 & 0.007038097 & 2.612736 \\
\hline & & 0.99 & 7.564561 & 0.0625094 & 9.347866 \\
\hline & \multirow{4}{*}{2} & 0.4 & 1.028419 & 0.00826265 & 1.028911 \\
\hline & & 0.7 & 1.164313 & 0.0068932 & 1.167031 \\
\hline & & 0.95 & 2.559845 & 0.00707358 & 2.629815 \\
\hline & & 0.99 & 7.524884 & 0.06157131 & 9.365334 \\
\hline & \multirow{4}{*}{5} & 0.4 & 1.029756 & 0.00819661 & 1.030243 \\
\hline & & 0.7 & 1.161748 & 0.00703535 & 1.164477 \\
\hline & & 0.95 & 2.54889 & 0.0072963 & 2.620243 \\
\hline & & 0.99 & 7.54267 & 0.06019454 & 9.335837 \\
\hline
\end{tabular}


Table 3: MSE of AR (1) with constant when presenting missing from (25 to 30) \%

\begin{tabular}{|c|c|c|c|c|c|}
\hline \multirow[b]{2}{*}{$\mathbf{n}$} & \multirow[b]{2}{*}{$\eta$} & \multirow[b]{2}{*}{$\rho$} & \multicolumn{3}{|c|}{ Methods } \\
\hline & & & OLS & MOLS & YW \\
\hline \multirow{12}{*}{20} & \multirow{4}{*}{$\mathbf{0}$} & 0.4 & 0.989734 & 0.3630099 & 1.003201 \\
\hline & & 0.7 & 1.16342 & 0.2793209 & 1.2121 \\
\hline & & 0.95 & 2.046242 & 0.526814 & 6.227486 \\
\hline & & 0.99 & 3.522668 & 0.9450221 & 303.5422 \\
\hline & \multirow{4}{*}{2} & 0.4 & 0.986509 & 0.3559574 & 0.9995428 \\
\hline & & 0.7 & 1.157195 & 0.2765698 & 1.203877 \\
\hline & & 0.95 & 2.058793 & 0.5247518 & 6.672372 \\
\hline & & 0.99 & 3.544081 & 0.9450047 & 322.6182 \\
\hline & \multirow{4}{*}{5} & 0.4 & 0.9830455 & 0.3581278 & 0.9959527 \\
\hline & & 0.7 & 1.154696 & 0.2784056 & 1.203093 \\
\hline & & 0.95 & 2.06056 & 0.5184065 & 6.136531 \\
\hline & & 0.99 & 3.519206 & 0.9365839 & 328.6834 \\
\hline \multirow{12}{*}{50} & \multirow{4}{*}{$\mathbf{0}$} & 0.4 & 1.021325 & 0.1105151 & 1.025451 \\
\hline & & 0.7 & 1.219952 & 0.07860798 & 1.241476 \\
\hline & & 0.95 & 2.680779 & 0.1423166 & 3.177464 \\
\hline & & 0.99 & 4.699261 & 0.4456397 & 17.98212 \\
\hline & \multirow{4}{*}{2} & 0.4 & 1.02674 & 0.1127874 & 1.030933 \\
\hline & & 0.7 & 1.219617 & 0.07717804 & 1.240231 \\
\hline & & 0.95 & 2.681446 & 0.1482986 & 3.197909 \\
\hline & & 0.99 & 4.749971 & 0.435359 & 18.19253 \\
\hline & \multirow{4}{*}{5} & 0.4 & 1.027967 & 0.1131732 & 1.032161 \\
\hline & & 0.7 & 1.219478 & 0.07704651 & 1.240723 \\
\hline & & 0.95 & 2.666624 & 0.1438715 & 3.175342 \\
\hline & & 0.99 & 4.679432 & 0.4303502 & 15.38143 \\
\hline \multirow{12}{*}{100} & \multirow{4}{*}{$\mathbf{0}$} & 0.4 & 1.037259 & 0.04349907 & 1.039462 \\
\hline & & 0.7 & 1.24749 & 0.03766628 & 1.259853 \\
\hline & & 0.95 & 3.138684 & 0.05548763 & 3.509958 \\
\hline & & 0.99 & 7.046477 & 0.2578023 & 12.24296 \\
\hline & \multirow{4}{*}{2} & 0.4 & 1.037563 & 0.04437655 & 1.039803 \\
\hline & & 0.7 & 1.251381 & 0.03887541 & 1.264006 \\
\hline & & 0.95 & 3.127201 & 0.05437775 & 3.49849 \\
\hline & & 0.99 & 6.958483 & 0.2564259 & 12.3649 \\
\hline & \multirow{4}{*}{5} & 0.4 & 1.041536 & 0.04389702 & 1.043784 \\
\hline & & 0.7 & 1.247821 & 0.03811179 & 1.260122 \\
\hline & & 0.95 & 3.144592 & 0.0539822 & 3.50933 \\
\hline & & 0.99 & 7.086264 & 0.2540915 & 12.46207 \\
\hline
\end{tabular}




\begin{tabular}{|c|c|c|c|c|c|}
\hline \multirow[b]{2}{*}{$\mathbf{n}$} & \multirow[b]{2}{*}{$\eta$} & \multirow[b]{2}{*}{$\boldsymbol{\rho}$} & \multicolumn{3}{|c|}{ Methods } \\
\hline & & & OLS & MOLS & YW \\
\hline \multirow{12}{*}{250} & \multirow{4}{*}{$\mathbf{0}$} & 0.4 & 1.0473 & 0.01093144 & 1.048205 \\
\hline & & 0.7 & 1.25368 & 0.01132473 & 1.258704 \\
\hline & & 0.95 & 3.397033 & 0.01301089 & 3.551554 \\
\hline & & 0.99 & 10.48604 & 0.09989088 & 14.33149 \\
\hline & \multirow{4}{*}{2} & 0.4 & 1.049174 & 0.01089173 & 1.050059 \\
\hline & & 0.7 & 1.257273 & 0.01134291 & 1.262369 \\
\hline & & 0.95 & 3.390354 & 0.01259854 & 3.543414 \\
\hline & & 0.99 & 10.44989 & 0.09749443 & 14.15096 \\
\hline & \multirow{4}{*}{5} & 0.4 & 1.046947 & 0.01102443 & 1.047841 \\
\hline & & 0.7 & 1.256377 & 0.01096677 & 1.261484 \\
\hline & & 0.95 & 3.373794 & 0.01272019 & 3.527032 \\
\hline & & 0.99 & 10.37268 & 0.09832786 & 14.09242 \\
\hline
\end{tabular}

\section{References}

1. Abdelwahab M.M. (2016). On Parameter estimation of time series models with missing observations. Unpublished $\mathrm{PhD}$ thesis. Cairo University Egypt.

2. Abdelwahab, M. M. and Issa M. K. A, (2019). Forms of the moments of AR (P) model with missing observations. The Egyptian statistical journal 63(1), pp. 39-44.

3. Bloomfield, P. (1970). Spectral analysis with randomly missing observations. Journal of the Royal Statistical Society Series B, 32, pp.369380.

4. Dunsmuir, W. (1981). Estimation for stationary time series when data are irregularly spaced or missing. In Applied Time Series Analysis II, (ed. David F. Findley). New York: Academic Press, pp.609-649.

5. Dunsmuir, W. and Robinson, P. M. (1981a). Asymptotic theory for time series containing missing and amplitude modulated observations. Sankhyā: The Indian Journal of Statistics, Series A, 43(3),pp. 260-281.

6. Dunsmuir, W. and Robinson, P. M. (1981b). Parametric estimators for stationary time series with missing observations. Advances in Applied Probability, 13(1), pp.129-146.

7. El-Sayed, S. M., El-Sheikh, A. A., and Abdelwahab, M. M. (2016). Estimation of $\mathrm{AR}(1)$ models with missing values. Advances and Applications in Statistics 49 (6), pp. 485-492.

8. Enany, M.A., Issa M.K. and Gad A.A. (2020). Maximum likelihood estimation of unconditional AR (1) model with missing observations. Working paper. Zagazig University: Egypt.

9. Issa M. K. A and Abdelwahab, M. M. (2020). Estimation of AR(1) panel data model with missing obsevations. Advances and Applications in Statistics 63 (1),pp, 109-117.

10. Jones, R. H. (1962). Spectral analysis with regularly missed observations. The Annals of Mathematical Statistics, 33,pp. 455-461. 
11. Little, R. J. A. and Rubin, D. B. (2002). Statistical analysis with missing data, $2^{\text {nd }}$ edn. John Wiley $\&$ Sons.

12. Parzen, E. (1963), On spectral analysis with missing observations and amplitude modulation. Sankhya Series A, 25, pp. 383-392.

13. Parzen, E. (ed) (1983). Proceedings of time series analysis of irregularly observed data. Lecture Notes in Statistics, New York: Springer Verlag.

14. Saadatmand, A., Nematollahi, A. R., and Sadooghi-Alvandi, S. M. (2017). On the estimation of missing values in AR (1) model with exponential innovations. Communications in Statistics-Theory and Methods, 46(7), pp.3393-3400.

15. Scheinok, P. A. (1965). Spectral analysis with randomly missed observations: The binomial case. The Annals of Mathematical Statistics, 36,pp 971-977.

16. Takeuchi, K. (1995). A comment on "Recent Development of Economic Data Analysis" at the $63^{\text {rd }}$ Annual Meeting of Japan Statistical Society

17. Yajima, Y. and Nishino, H. (1999). Estimation of the autocorrelation function of a stationary time series with missing observations. Sankhyā: The Indian Journal of Statistics, Series A ,61(2),pp. 189-207.

18. Youssef, A. H. (2006) . A performance of alternative predictors for the unit root process. interstatstat journal, USA 